\title{
Tuberculin Skin Test in Spondyloarthritis: Overestimated if Rheumatoid Arthritis Guidelines for Latent Tuberculosis Are Used?
}

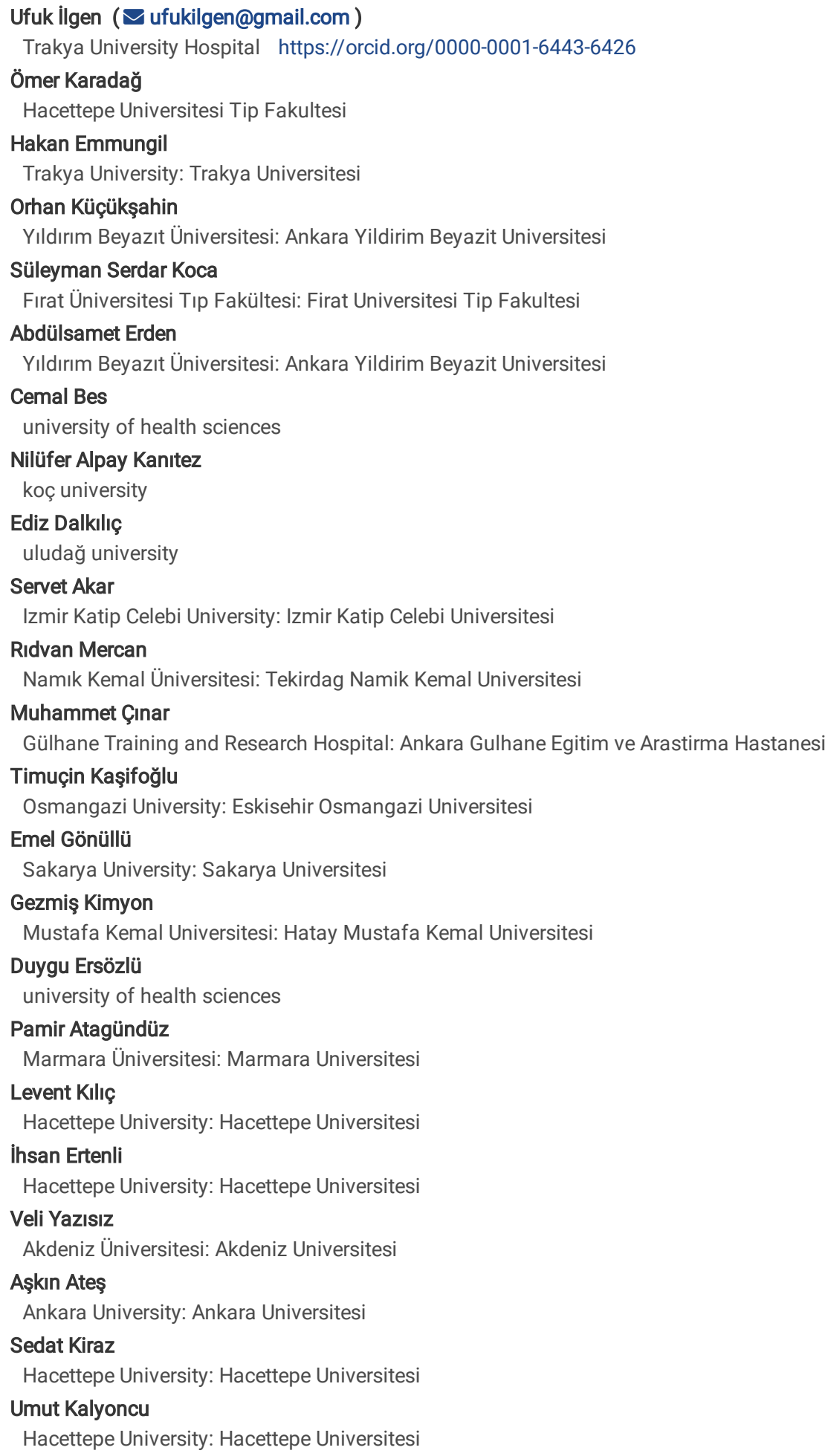

Keywords: interferon-y release assay, QuantiFERON®-TB Gold In-Tube, latent tuberculosis infection, rheumatoid arthritis, spondyloarthritis, tuberculin skin test, tuberculosis 
Posted Date: December 8th, 2020

DOl: https://doi.org/10.21203/rs.3.rs-121794/v1

License: (c) (1) This work is licensed under a Creative Commons Attribution 4.0 International License. Read Full License

Version of Record: A version of this preprint was published at Rheumatology International on April 29th, 2022. See the published version at https://doi.org/10.1007/s00296-022-05134-z. 


\section{Abstract}

Background: Several societies published recommendations for latent tuberculosis infection (LTBI) screening before biological and targeted synthetic diseasemodifying anti-rheumatic drugs (DMARDs) in rheumatoid arthritis (RA) but not for other inflammatory arthritides such as spondyloarthritis (SpA). Using RA guidelines could result in overestimating Tuberculin Skin Test (TST) positivity in SpA. This study aimed to compare the distribution of TST results in SpA and RA patients along with comparison in terms of QuantiFERON®-TB Gold In-Tube (QFT-GIT) test in a Bacillus Calmette-Guérin-vaccinated population.

Methods: Adult RA ( $n=206)$ and SpA ( $n=392)$ patients from the TReasure database who had both TST and QFT-GIT prior to initiation of biological and targeted synthetic DMARDs were included in the study. Demographic and disease characteristics along with pre-biologic DMARD and steroid use were recorded. The distribution of TST and performance with respect to QFT-GIT were compared between RA and SpA groups.

Results: Pre-biologic conventional DMARD and steroid use was higher in the RA group. TST positivity rates were $44.2 \%$ in RA and $69.1 \%$ in SpA for a 5 mm cutoff $(p<0.001)$. QFT-GIT positivity was slighlty higher in the SpA group ( $15 \%$ vs $8.9 \%, p=0.075)$. QFT-GIT positivity and disease category independently predicted a $5 \mathrm{~mm}$ or higher TST. The two tests poorly agreed in both groups at a TST cut-off of $5 \mathrm{~mm}$ ( $\kappa<0.1$ for both groups). Increasing the TST cut-off only slightly increased the agreement between the two tests.

Conclusions: TST positivity was more pronounced in SpA compared to that in RA and this was not explainable by pre-biologic DMARD and steroid use. The agreement of TST with QFT-GIT was poor in both groups. Using a $5 \mathrm{~mm}$ TST cut-off for both diseases could result in overestimating LTBI in SpA.

\section{Background}

Screening for and the treatment of latent tuberculosis infection (LTBI) is recommended in inflammatory arthritis patients prior to biological and targeted synthetic disease-modifying anti-rheumatic drugs (DMARDs), particularly the tumor necrosis factor- $a(T N F-a)$ inhibitors $[1,2]$. Screening and treatment strategies for LTBI differ across the world because of the epidemiological and economic reasons for which many regional guidelines exist in rheumatology practice [2-8].

Tuberculin skin test (TST) has been used for more than a century to detect infection with Mycobacterium tuberculosis. Potential false-positive results in Bacillus Calmette-Guérin (BCG)-vaccinated or non-tuberculous Mycobacteria (NTM)-infected people, interobserver variability, and false-negative results in immunocompromised patients are the major disadvantages of this test along with a need to interpret the test result according to the individual situation [1, 911]. Interferon-y (IFN-Y) release assays (IGRAs), QuantiFERON®-TB Gold In-Tube (QFT-GIT) and T-SPOT®. TB, are relatively new tests to detect latent infection with Mycobacterium tuberculosis and depend on the measurement of IFN- $\mathrm{y}$ produced by T lymphocytes incubated with Mycobacterium tuberculosis antigens. They are not affected by latent infections by most NTMs and BCG vaccination [1, 9, 12]. Both TST and IGRAs were mostly reported to have comparable sensitivity and specificity to detect LTBI in non-immunocompromised hosts, and either test may be used [1, 12-16]. However, recommendations for the preferential use of IGRAs over TST exist based on the reports with more accurate results by IGRAs [9, 17-19]. Conflicting results on the performance of IGRAs or combination tests (both TST and an IGRA or sequential testing according to an initial TST or IGRA) compared to TST alone have been reported in Human Immunodeficiency Virus (HIV)-uninfected immunocompromised adults [9, 20-28] but IGRAs, conditionally combined with a TST, are increasingly being recommended to screen LTBI particularly in BCG-vaccinated patients [3, 5-7, 12].

Although treatment with biological and targeted synthetic DMARDs itself puts patients with LTBI into an increased risk of tuberculosis reactivation (also called disease progression) $[1,9,12,25]$, the interpretation of the LTBI screening with TST before initiation of these drugs may not necessarily be the same in different patient groups with inflammatory arthritis since the degree of immunosuppression, mainly determined by the drugs used, comorbid diseases, and rheumatic disease itself, is not the same. Current guidelines and society recommendations for the screening and treatment of LTBI before biological and targeted synthetic DMARDs do not distinguish patients with different rheumatic diseases from each other [2, 5, 8]. Several societies make specific recommendations for LTBI screening or refer to local tuberculosis guidelines before biological and targeted synthetic DMARDs in rheumatoid arthritis (RA) [3, $4,6,7$ ] but not other inflammatory arthritides such as spondyloarthritis (SpA) requiring biological and targeted synthetic DMARDs [26-28]. The European League Against Rheumatism (EULAR) has also no recommendation regarding LTBI in any rheumatic disease.

Since the TST or IGRAs are not superior to each other in assessing LTBI in patients with rheumatic diseases, both could be ordered. The aim of this study was to compare the distribution of TST along with comparison in terms of QFT-GIT in RA and SpA patients who were candidates for biological and targeted synthetic DMARDs in a BCG-vaccinated population.

\section{Methods}

Patients and Design: TReasure is a web-based database to which users connect through a URL (https://www.trials-network.org/treasure) with their unique identifier and passwords provided for data entry and access. TReasure records demographic and clinical features, comorbidities, radiology and laboratory results, measures of disease activity, and treatment data of inflammatory rheumatic diseases such as RA and SpA [29]. Patients older than 18 years of age, with a diagnosis of RA or SpA, fulfilling 2010 American College of Rheumatology (ACR)/EULAR [30] and Assessment of Spondyloarthritis International Society (ASAS) criteria [31] were initially screened. 2690 RA and 4995 SpA patients were identified by the end of March 2019.1091 (40.6\%) and 1413 (52.5\%) patients in RA and 2377 (47.6\%) and 2509 (50.2\%) patients in the SpA group underwent testing with TST and QFT-GIT, respectively. 241 (9\%) and 439 (8.8\%) patients had both TST and QFT-GIT in RA and SpA groups. 35 RA and 47 SpA patients were excluded due to the presence or history of active tuberculosis, HIV infection, solid organ or hematopoietic stem cell transplantation, diabetes, chronic kidney disease, chronic liver disease, chronic obstructive pulmonary disease or persistent asthma, or malignancy. Finally, 206 RA and 392 SpA patients who had both TST and QFT-GIT were recruited for further analysis. 
Demographic and disease-related features including age, sex, education status, smoking status, disease duration, systemic steroid and conventional DMARD use prior to initiation of biologic and targeted synthetic DMARDs were identified retrospectively from the database along with BCG vaccination history, presence of a BCG scar, TST (in millimeters) and QFT-GIT (positive, negative, and indeterminate) results, and LTBI treatment based on the physician's decision. RA and SpA study groups were compared in terms of demographic and disease-related features, BCG vaccination status, TST and QFT-GIT results, and LTBI treatment rates. Comparison of the study groups with entire RA and SpA patients was provided in the additional file 1. Written informed consent was obtained from each patient as per the registry protocol.

TST and QFT-GIT: TST has traditionally been performed in Tuberculosis Dispensaries and Chest Diseases Departments of hospitals in Turkey in a standardized way according to the national tuberculosis guidelines [32]. Briefly, $0.1 \mathrm{~mL} 5$-tuberculin unit purified protein derivative (PPD) is administered intradermally in the forearm according to the Mantoux method. The largest induration diameter is measured 48-72 hours later by an expert and reported. QFTGIT (Cellestis Ltd, Carnegie, Victoria, Australia) test is available in many public and private hospitals and laboratories and increasingly used in Turkey. It is performed according to the manufacturer's instructions.

Statistical Analysis: PASW Statistics v.18.0 (SPSS Inc, Chicago, IL, USA) was used for the statistical analyses. Data were expressed as numbers with percentages for the categorical variables and means \pm standard deviations for the continuous ones. Categorical data were compared by using chi-square or Fisher's exact tests. Distributions of the continuous data were analyzed by histograms and tested for normality by Lilliefors-corrected Kolmogorov-Smirnov test. Continuous data were compared using the t-test or Mann-Whitney U test according to the distribution. Percent agreement of TST with QFT-GIT and Cohen's kappa coefficients were provided in RA and SpA groups separately. Multiple logistic regression analyses were performed with the potential predictors of TST positivity. Odds ratios (ORs) with $95 \%$ confidence intervals were calculated for risk assessments. $p<0.05$ was considered statistically significant.

\section{Results}

Mean disease duration of RA and SpA patients were $11.8 \pm 8$ and $8.7 \pm 6$ years, respectively. Demographic data, disease-related features, TST and QFT-GIT results prior to initiation of biological and targeted synthetic DMARDs and LTBI treatment rates were given in Table 1. Of 135 RA and 251 SpA patients questioned, $94.4 \%$ and $88.8 \%$ recalled a previous BCG vaccination ( $p=0.051) .87 / 89(97.8 \%)$ and 172/182 (94.5\%) patients in RA and SpA groups, respectively, checked for the presence of a BCG scar, had at least one scar as expected due to the national immunization program ( $p=0.348)$. The mean TST result was lower in RA compared to that in SpA patients $(5.7 \pm 5.8 \mathrm{vs}$. $9.3 \pm 6.4 \mathrm{~mm}, \mathrm{p}<0.001)$. The distribution of TST in study groups was represented in Fig. 1. The rates of positive TST at 5,10 , and $15 \mathrm{~mm}$ cut-off values were significantly higher in the SpA group (Table 1). QFT-GIT positivity rate was slightly higher in the SpA (15.1\%) compared to the RA group (9.7\%) but the difference was not statistically significant $(\mathrm{OR}=1.64$ [0.96-2.82], $\mathrm{p}=0.075)$. The treatment rate of latent tuberculosis was also higher in the SpA group (OR = 1.88 [1.33-2.64]) (Table 1). 
Table 1

Demographic data, disease-related features, TST and QFT-GIT results prior to initiation of biological and targeted synthetic DMARDs, and LTBI treatment rates

\begin{tabular}{|c|c|c|c|c|c|c|}
\hline & & $\mathbf{n}$ & RA & $\mathbf{n}$ & SpA & $\mathbf{p}$ \\
\hline Female sex, $\mathrm{n}(\%)$ & & 206 & $160(77.7)$ & 392 & $154(39.3)$ & $<0.001$ \\
\hline Age, years & & 206 & $49 \pm 15$ & 392 & $43 \pm 11$ & $<0.001$ \\
\hline \multirow[t]{2}{*}{ Education status, n(\%) } & Primary or lower & 201 & $91(45.2)$ & 379 & $85(22.4)$ & $<0.001$ \\
\hline & Higher education & & $110(54.8)$ & & $294(77.6)$ & \\
\hline \multirow[t]{3}{*}{ Smoking status, n(\%) } & Never smoked & 202 & $130(64.4)$ & 369 & $154(41.7)$ & $<0.001$ \\
\hline & Ex-smoker & & 35 (17.3) & & $63(17.1)$ & \\
\hline & Active smoker & & $37(18.3)$ & & $152(41.2)$ & \\
\hline Disease duration, years & & 202 & $11.8 \pm 8$ & 392 & $8.7 \pm 6$ & $<0.001$ \\
\hline Systemic steroid use, $\mathrm{n}(\%)$ & & 206 & $113(54.9)$ & 392 & 73 (18.6) & $<0.001$ \\
\hline \multirow[t]{5}{*}{ cDMARD use, $n(\%)$} & & 206 & $172(83.5)$ & 392 & $245(62.5)$ & $<0.001$ \\
\hline & Methotrexate & & $137(66.5)$ & & $89(22.7)$ & $<0.001$ \\
\hline & Hydroxychloroquine & & $93(45.1)$ & & $38(9.7)$ & $<0.001$ \\
\hline & Sulfasalazine & & $110(53.4)$ & & $217(55.4)$ & 0.647 \\
\hline & Leflunomide & & $76(36.9)$ & & $18(4.6)$ & $<0.001$ \\
\hline TST, mm & & 206 & $5.7 \pm 5.8$ & 392 & $9.3 \pm 6.4$ & $<0.001$ \\
\hline \multirow[t]{4}{*}{ TST, n(\%) } & $=0 \mathrm{~mm}$ (complete anergy) & 206 & $59(28.6)$ & 392 & $43(11.0)$ & $<0.001$ \\
\hline & $\geq 5 \mathrm{~mm}$ & & $91(44.2)$ & & $271(69.1)$ & $<0.001$ \\
\hline & $\geq 10 \mathrm{~mm}$ & & $38(18.4)$ & & $154(39.3)$ & $<0.001$ \\
\hline & $\geq 15 \mathrm{~mm}$ & & $16(7.8)$ & & $60(15.3)$ & 0.009 \\
\hline \multirow[t]{3}{*}{ QFT-GIT, n(\%) } & Positive & 206 & $20(9.7)$ & 392 & $59(15.1)$ & 0.075 \\
\hline & Negative & & $185(89.8)$ & & $333(84.9)$ & \\
\hline & Indeterminate & & $1(0.5)$ & & - & \\
\hline LTBI treatment, n(\%) & & 206 & $92(44.7)$ & 382 & $230(60.2)$ & $<0.001$ \\
\hline
\end{tabular}

Male sex, higher education, and smoking were more frequent in patients with a TST of $5 \mathrm{~mm}$ or higher compared to those with TST less than $5 \mathrm{~mm}$, if RA and SpA groups were collated. The mean age was lower and systemic steroid, methotrexate, and leflunomide use were less frequent in TST $\geq 5$ mm group as well (Table 2). 
Table 2

Factors associated with TST positivity for a $5 \mathrm{~mm}$ cut-off value

\begin{tabular}{|c|c|c|c|c|c|c|}
\hline & & $\mathrm{n}$ & $\mathrm{TST}<5 \mathrm{~mm}$ & $\mathbf{n}$ & $\mathrm{TST} \geq 5 \mathrm{~mm}$ & $\mathbf{p}$ \\
\hline Female sex, n(\%) & & 236 & $152(64.4)$ & 362 & $162(44.8)$ & $<0.001$ \\
\hline Age, years & & 236 & $46.3 \pm 14.1$ & 362 & $43.9 \pm 12$ & $<0.001$ \\
\hline \multirow[t]{2}{*}{ Education status, $\mathrm{n}(\%)$} & Primary or lower & 226 & $85(37.6)$ & 354 & $91(25.7)$ & 0.002 \\
\hline & Higher education & & $141(62.4)$ & & $263(74.3)$ & \\
\hline \multirow[t]{3}{*}{ Smoking status, n(\%) } & Never smoked & 224 & $132(58.9)$ & 347 & $152(43.8)$ & 0.001 \\
\hline & Ex-smoker & & $35(15.6)$ & & $63(18 . .2)$ & \\
\hline & Active smoker & & $57(25.4)$ & & $132(38)$ & \\
\hline Systemic steroid use, $\mathrm{n}(\%)$ & & 236 & 89 (37.7) & 362 & $97(26.8)$ & 0.007 \\
\hline \multirow[t]{5}{*}{ cDMARD use, $n(\%)$} & & 236 & $178(75.4)$ & 362 & $239(66)$ & 0.014 \\
\hline & Methotrexate & & $103(43.6)$ & & $123(34)$ & 0.017 \\
\hline & Hydroxychloroquine & & $57(24.2)$ & & $74(20.4)$ & 0.284 \\
\hline & Sulfasalazine & & $133(56.4)$ & & $194(53.6)$ & 0.507 \\
\hline & Leflunomide & & $54(22.9)$ & & $40(11)$ & $<0.001$ \\
\hline \multirow[t]{2}{*}{ Disease category, $n(\%)$} & Rheumatoid arthritis & 236 & $115(48.7)$ & 362 & $91(25.1)$ & $<0.001$ \\
\hline & Spondyloarthritis & & $121(51.3)$ & & $271(74.9)$ & \\
\hline \multirow[t]{3}{*}{ QFT-GIT, n(\%) } & Positive & 236 & $10(4.2)$ & 362 & $69(19.1)$ & $<0.001$ \\
\hline & Negative & & $225(95.4)$ & & $293(80.9)$ & \\
\hline & Indeterminate & & $1(0.4)$ & & - & \\
\hline
\end{tabular}

Multiple logistic regression analysis with the covariates age, sex, education, smoking, systemic steroid, methotrexate, and leflunomide use, and disease category identified the disease category as the only significant predictor of a TST $\geq 5 \mathrm{~mm}$ (the OR of a positive test was 2.05 [1.33-3.17] in SpA with reference to RA, $p=0.001$ ). The relationship between the disease category and TST positivity was significant even after the addition of the QFT-GIT positivity to the covariates (Table 3).

Table 3

Multiple logistic regression analysis for TST positivity for a $5 \mathrm{~mm}$ cut-off value

\begin{tabular}{|llll|}
\hline & Odds ratio & $95 \%$ Cl & p \\
\hline Male sex & 1.43 & $0.97-2.11$ & 0.074 \\
\hline Age & 0.99 & $0.98-1.01$ & 0.640 \\
\hline Higher education & 1.25 & $0.83-1.88$ & 0.284 \\
\hline Ever-smoking & 1.24 & $0.86-1.80$ & 0.258 \\
\hline Systemic steroid use & 0.85 & $0.55-1.31$ & 0.470 \\
\hline Methotrexate use & 1.20 & $0.79-1.85$ & 0.393 \\
\hline Leflunomide use & 0.74 & $0.43-1.27$ & 0.270 \\
\hline Disease category (SpA) & 2.03 & $1.31-3.14$ & $\mathbf{0 . 0 0 2}$ \\
\hline QFT-GIT positivity & 2.56 & $1.41-4.65$ & 0.002 \\
\hline CI = confidence interval; TST = tuberculin skin test; QFT-GIT = Quantiferon ${ }^{\circledR}$-TB Gold In-Tube; SpA = spondyloarthritis \\
\hline
\end{tabular}

The distributions of TST according to the QFT-GIT status were quite different in RA and SpA groups (Fig. 1). TST results according to the QFT-GIT status for a $5 \mathrm{~mm}$ cut-off value were represented in Table 4 . The two tests poorly agreed with $\mathrm{k}$ coefficients of 0.02 and 0.08 in RA and SpA groups, respectively. Note that TST with a $5 \mathrm{~mm}$ cut-off value could detect only half of the QFT-GIT positive patients in RA and was positive in two-thirds of the QFT-GIT negative SpA patients (Table 4). Increasing the TST cut-off only slightly increased the agreement between the two tests (Additional file 2). 
Table 4

TST results according to the QFT-GIT status in study groups for a $5 \mathrm{~mm}$ cut-off value

\begin{tabular}{|c|c|c|c|c|}
\hline & RA & & SpA & \\
\hline & $\begin{array}{l}\text { QFT-GIT negative } \\
(\mathrm{n}=185)\end{array}$ & $\begin{array}{l}\text { QFT-GIT positive } \\
(n=20)\end{array}$ & $\begin{array}{l}\text { QFT-GIT negative } \\
(n=333)\end{array}$ & $\begin{array}{l}\text { QFT-GIT positive } \\
(n=59)\end{array}$ \\
\hline $\mathrm{TST}<5 \mathrm{~mm}$ & $104(56.2 \%)$ & $10(50 \%)$ & $113(33.9 \%)$ & $8(13.5 \%)$ \\
\hline \multirow[t]{3}{*}{$\mathrm{TST} \geq 5 \mathrm{~mm}$} & $81(43.8 \%)$ & $10(50 \%)$ & $220(66.1 \%)$ & $51(86.5 \%)$ \\
\hline & Agreement $=55.6 \%$ & & Agreement $=41.8 \%$ & \\
\hline & Cohen's $\mathrm{K}=0.02$ & & Cohen's $\mathrm{K}=0.08$ & \\
\hline
\end{tabular}

\section{Discussion}

We were able to show that TST positivity rate was significantly higher in SpA patients compared to that of RA patients prior to initiation of biological and targeted synthetic DMARDs, although BCG scar rates were similar and QFT-GIT positivity rates were only slightly different. Although the smoking rate was higher, and systemic steroid and conventional DMARD use were less frequent in SpA compared to RA, a higher rate of TST positivity was not attributable to those (Table 3).

Treatment with immunosuppressive medications has been known to block the immune response against tuberculin and Mycobacterium tuberculosis-specific antigens to some degree and may be responsible for false-negative TST and QFT-GIT results [11, 33]. A higher rate of complete cutaneous anergy and slightly lower QFT-GIT positivity in RA compared to the SpA group in the present study may be caused by more frequent use of systemic steroids and conventional DMARDs in RA. The potential impact of intrinsic immune dysregulation in rheumatic diseases on LTBI and screening tests was not evaluated before. But diminished immune response against microbes and vaccines was attributed not only to immunosuppressive medications but the disease itself in patients with RA [34-36].

Conflicting results on the performance of IGRAs compared to TST in terms of sensitivity and specificity to detect LTBI have been reported in immunocompromised adults without HIV infection [20-24]. The principal reason for that is the lack of a gold standard test for LTBI, which is, by definition, the presence of an immune response - assumed to be caused by a previous sensitization - against Mycobacterium tuberculosis antigens with no evidence of active tuberculosis [1]. It is not a direct microbiological diagnosis, and false-positive and -negative results are of great concern both by TST and IGRAs [1, 11, 12]. It is also difficult to evaluate the progression to active tuberculosis in immunocompromised patients tested by TST and IGRAs comparatively since patients with positive results of either test are usually given treatment due to a high risk of reactivation. According to the present and two previous studies [20, 24], increasing the TST cut-off value slightly improved the agreement between the two tests but to a moderate level at most. So, TST - QFT-GIT disagreement in the immunocompromised adult population without HIV infection does not seem to be caused primarily by a cut-off issue. BCG vaccination is a well-known factor for false-positive TST results and a potential reason to use IGRAs to detect LTBI [3,5-7, 12] but cannot explain the discrepant study results conducted in BCG-vaccinated patient groups [20-24]. A possible reason why studies report different TST - QFT-GIT agreement rates in BCG-vaccinated patients may be the difference in the patient groups (i.e. patient groups with different diseases) and the degree of immunosuppression of the study groups. According to a meta-analysis of long-term extension studies, not only TST - QFT-GIT agreement but the actual tuberculosis risk was also different in different rheumatic diseases including RA and SpA independent of the treatment with biologics [37]. Treatment with TNF-a inhibitors increased the risk of tuberculosis in both RA and SpA but to a higher level in RA [37]. Not so unexpectedly, studies conducted in different patient groups, such as inflammatory bowel disease patients under treatment with various immunosuppressive agents, TNF-a inhibitor-scheduled patients with rheumatic diseases under DMARDs, and solid organ transplantation candidates with no immunosuppressive medication use reported different agreement rates between TST and QFT-GIT [20, 24]. Different TST results were reported even in psoriasis and psoriatic arthritis patients despite similar QFT-GIT results [38]. Different agreement rates between TST and QFT-GIT in RA and SpA in the present study may represent an example of this situation. To overcome the effect of conventional DMARD and steroid treatment on screening tests, the Australian Rheumatology Association suggests screening LTBI at the initial diagnosis of inflammatory arthritis [5]. Anyway, candidates for biological and targeted synthetic DMARDs have traditionally been screened in the same way regardless of their underlying rheumatic disease, although both the tuberculosis progression (reactivation) rates and the screening test results may differ. It should additionally be stated that TST procedures with different types and units of tuberculin products in different countries may also contribute to discrepant study results [11, 20-24].

In correlation with TST positivity rates for a $5 \mathrm{~mm}$ cut-off as suggested by the Turkish Society for Rheumatology, Turkish Thoracic Society, and Ministry of Health [8], LTBI treatment rates were higher in SpA compared to RA group (Table 1). There lies a paradox here. RA patients, who are more immunosuppressed and more prone to tuberculosis reactivation, were given less LTBI treatment since they had lower TST positivity compared to SpA patients. The opposite seems true for SpA patients. This particular point implies the necessity of studies in separate disease groups rather than pre-biologic patient pools.

IGRA-only and combined test approaches were proved effective and safe particularly to reduce overtreatment with antituberculosis drugs in immunocompromised and BCG-vaccinated patients [21,39-42] but debate exists on this topic [15, 22, 23, 33]. In a longitudinal study of inflammatory arthritis patients comparing different baseline LTBI screening strategies before TNF-a inhibitors in a high tuberculosis burden BCG-vaccinated population, incidence rates of active tuberculosis, after a mean exposure of $4 \pm 2.4$ years to TNF-a inhibitors, were 1348.0, 862.1, and 540.2 cases/100000 patient-years in TST (cutoff $\geq 10 \mathrm{~mm}$ ), TST (cut-off $\geq 5 \mathrm{~mm}$ ), and QFT groups, respectively, although the difference was not found statistically significant [43]. Cost-effectiveness and antituberculosis drug-related toxicity are also important concerns regarding LTBI screening and treatment strategies but beyond the scope of this study.

Page $7 / 11$ 
There are several limitations of this study. This was a cross-sectional study and tuberculosis progression (reactivation) rates were not available. TST and QFTGIT were performed in different centers and it was not known which test was performed first. Test intervals were also not known. Since BCG vaccination is routine in Turkey, the missing data on BCG scar and vaccination history do not seem to cause confusion to interpret the study results. There were some differences in age, education status, pre-biologic systemic steroid and conventional DMARD use, and TST results between the study groups and the entire RA and SpA populations (Additional file 1). But these were not thought to have a major impact on the main results. Overall, this study adds valuable information to the relevant field regarding the difference in the performance of LTBI screening tests in RA and SpA.

\section{Conclusions}

TST positivity was more pronounced in SpA compared to RA and this was not explainable by pre-biologic DMARD and steroid use. The agreement of TST with QFT-GIT for latent tuberculosis was poor and increasing the TST cut-off only slightly increased the agreement between the two tests. Using a 5 mm TST cutoff for both diseases could result in overestimating LTBI in SpA.

\section{Abbreviations}

ACR: American College of Rheumatology; ASAS: Assessment of Spondyloarthritis International Society; BCG: Bacillus Calmette-Guérin; DMARD: DiseaseModifying Anti-Rheumatic Drug; EULAR: European League Against Rheumatism; HIV: Human Immunodeficiency Virus; IFN: Interferon; IGRA: Interferon-Y Release Assay, LTBI: Latent Tuberculosis Infection; NTM: Non-Tuberculous Mycobacteria; QFT-GIT: QuantiFERON®-TB Gold In-Tube Test; RA: Rheumatoid Arthritis; SpA: Spondyloarthritis; TNF: Tumor Necrosis Factor; TST: Tuberculin Skin Test

\section{Declarations}

\section{Ethics approval and consent to participate:}

Written informed consent was obtained from each patient regarding the use of clinical data for research purposes. The study was in accordance with the 2013 amendment of the Helsinki declaration. Ethical approval was obtained from Hacettepe University Institutional Review Board (KA17/058, May 2017) and Ministry of Health of Turkey (93189304-14.03.01, October 2017).

\section{Consent for publication:}

Not applicable.

\section{Availability of data and materials:}

The data underlying this article were accessed from the TReasure database (URL: https://www.trials-network.org/treasure). The derived data generated in this research will be shared on reasonable request to the corresponding author.

\section{Competing interests:}

The authors declare that they have no competing interests.

\section{Funding:}

None.

\section{Authors' contributions:}

Conception \& Design: U.I., H.E., U.K. Data acquisition \& Analysis: U.I., Ö.K., H.E., S.A., L.K., U.K. Data interpretation: U.I., Ö.K., H.E., O.K., S.S.K., A.E., C.B., N.A.K., E.D., S.A., R.M., M.C., T.K., E.G., G.K., D.E., P.A., L.K., I.E., V.Y., A.A., S.K., U.K. Drafting \& Critical review: U.I., Ö.K., H.E., O.K., S.S.K., A.E., C.B., N.A.K., E.D., S.A., R.M., M.C., T.K., E.G., G.K., D.E., P.A., L.K., I.E., V.Y., A.A., S.K., U.K. All authors read and approved the final manuscript.

\section{Acknowledgments:}

Not applicable.

\section{References}

1. World Health Organization. Latent tuberculosis infection - Updated and consolidated guidelines for programmatic management. Geneva, 2018. https://www.who.int/tb/publications/2018/latent-tuberculosis-infection/en/ Accessed 20.10.2020

2. lannone F, Cantini F, Lapadula G. Diagnosis of latent tuberculosis and prevention of reactivation in rheumatic patients receiving biologic therapy: international recommendations. J Rheumatol Suppl 2014; 91: 41-6. doi: 10.3899/jrheum.140101

3. Singh JA, Saag KG, Bridges SL Jr, AkI EA, Bannuru RR, Sullivan MC, et al. 2015 American College of Rheumatology Guideline for the Treatment of Rheumatoid Arthritis. Arthritis Rheumatol 2016; 68: 1-26. doi: 10.1002/art.39480

4. Koike R, Takeuchi T, Eguchi K, Miyasaka N; Japan College of Rheumatology. Update on the Japanese guidelines for the use of infliximab and etanercept in rheumatoid arthritis. Mod Rheumatol 2007; 17: 451-8. doi: 10.1007/s10165-007-0626-3 
5. Australian Rheumatology Association. Screening for Latent Tuberculosis Infection (LTBI) and its management in Inflammatory arthritis patients. 2018. https://rheumatology.org.au/gps/documents/SCREENINGFORLATENTTUBERCULOSISINFECTION_Jan18.pdf Accessed 20.10.2020

6. Parisi S, Bortoluzzi A, Sebastiani GD, Conti F, Caporali R, Ughi N, et al. The Italian Society for Rheumatology clinical practice guidelines for rheumatoid arthritis. Reumatismo 2019; 71: 22-49. doi: 10.4081/reumatismo.2019.1202

7. Bombardier C, Hazlewood GS, Akhavan P, Schieir O, Dooley A, Haraoui B, et al. Canadian Rheumatology Association recommendations for the pharmacological management of rheumatoid arthritis with traditional and biologic disease-modifying antirheumatic drugs: part II safety. J Rheumatol 2012; 39: 1583-602. doi: 10.3899/jrheum.120165

8. T.C. Sağlık Bakanlığı Türkiye Halk Sağlığı Kurumu. Anti-TNF kullanan hastalarda tüberküloz rehberi. Ankara, 2016. [Turkish] http://www.romatoloji.org/Dokumanlar/Site/ATKHTR.pdf Accessed 20.10.2020

9. Lewinsohn DM, Leonard MK, LoBue PA, Cohn DL, Daley CL, Desmond E, et al. Official American Thoracic Society/Infectious Diseases Society of America/Centers for Disease Control and Prevention Clinical Practice Guidelines: Diagnosis of Tuberculosis in Adults and Children. Clin Infect Dis 2017; 64: 111-5. doi: 10.1093/cid/ciw778

10. [No authors listed] Targeted tuberculin testing and treatment of latent tuberculosis infection. Am J Respir Crit Care Med 2000; 161: S221-47. doi: 10.1164/ajrccm.161.supplement_3.ats600

11. Lee E, Holzman RS. Evolution and current use of the tuberculin test. Clin Infect Dis 2002; 34: 365-70. doi: 10.1086/338149

12. Centers for Disease Control and Prevention. Updated guidelines for using Interferon Gamma Release Assays to detect Mycobacterium tuberculosis infection - United States, 2010. MMWR Recomm Rep 2010; 59: 1-25.

13. Mahomed H, Hawkridge T, Verver S, Abrahams D, Geiter L, Hatherill M, et al. The tuberculin skin test versus QuantiFERON TB Gold® in predicting tuberculosis disease in an adolescent cohort study in South Africa. PLoS One. 2011; 6: e17984. doi: 10.1371/journal.pone.0017984

14. Sharma SK, Vashishtha R, Chauhan LS, Sreenivas V, Seth D. Comparison of TST and IGRA in Diagnosis of Latent Tuberculosis Infection in a High TBBurden Setting. PLoS One. 2017; 12: e0169539. doi: 10.1371/journal.pone.0169539

15. Kampmann B, Whittaker E, Williams A, Walters S, Gordon A, Martinez-Alier N, et al. Interferon-gamma release assays do not identify more children with active tuberculosis than the tuberculin skin test. Eur Respir J 2009; 33: 1374-82. doi: 10.1183/09031936.00153408

16. Brock I, Weldingh K, Lillebaek T, Follmann F, Andersen P. Comparison of tuberculin skin test and new specific blood test in tuberculosis contacts. Am J Respir Crit Care Med 2004; 170: 65-9. doi: 10.1164/rccm.200402-2320C

17. Arend SM, Thijsen SF, Leyten EM, Bouwman JJ, Franken WP, Koster BF, et al. Comparison of two interferon-gamma assays and tuberculin skin test for tracing tuberculosis contacts. Am J Respir Crit Care Med 2007; 175: 618-27. doi: 10.1164/rccm.200608-10990C

18. Diel R, Loddenkemper R, Meywald-Walter K, Gottschalk R, Nienhaus A. Comparative performance of tuberculin skin test, QuantiFERON-TB-Gold In Tube assay, and T-Spot.TB test in contact investigations for tuberculosis. Chest 2009; 135: 1010-8. doi: 10.1378/chest.08-2048

19. Hill PC, Jeffries DJ, Brookes RH, Fox A, Jackson-Sillah D, Lugos MD, et al. Using ELISPOT to expose false positive skin test conversion in tuberculosis contacts. PLoS One 2007; 2: e183. doi: 10.1371/journal.pone.0000183

20. Erol S, Ciftci FA, Ciledag A, Kaya A, Kumbasar 00. Do higher cut-off values for tuberculin skin test increase the specificity and diagnostic agreement with interferon gamma release assays in immunocompromised Bacillus Calmette-Guérin vaccinated patients? Adv Med Sci 2018; 63: 237-41. doi: 10.1016/j.advms.2017

21. Jeong DH, Kang J, Jung YJ, Yoo B, Lee CK, Kim YG, et al. Comparison of latent tuberculosis infection screening strategies before tumor necrosis factor inhibitor treatment in inflammatory arthritis: IGRA-alone versus combination of TST and IGRA. PLoS One 2018; 13: e0198756. doi: 10.1371/journal.pone.0198756

22. Kim EY, Lim JE, Jung JY, Son JY, Lee KJ, Yoon YW, et al. Performance of the tuberculin skin test and interferon-gamma release assay for detection of tuberculosis infection in immunocompromised patients in a BCG-vaccinated population. BMC Infect Dis 2009; 9: 207. doi: 10.1186/1471-2334-9-207

23. Hanta I, Ozbek S, Kuleci S, Seydaoglu G, Ozyilmaz E. Detection of latent tuberculosis infection in rheumatologic diseases before anti-TNFa therapy: tuberculin skin test versus IFN-y assay. Rheumatol Int 2012; 32: 3599-603. doi: 10.1007/s00296-011-2243-x

24. Kurti Z, Lovasz BD, Gecse KB, Balint A, Farkas K, Morocza-Szabo A, et al. Tuberculin Skin Test and Quantiferon in BCG Vaccinated, Immunosuppressed Patients with Moderate-to-Severe Inflammatory Bowel Disease. J Gastrointestin Liver Dis 2015; 24: 467-72. doi: 10.15403/jgld.2014.1121.244.bcg

25. Ai JW, Ruan QL, Liu QH, Zhang WH. Updates on the risk factors for latent tuberculosis reactivation and their managements. Emerg Microbes Infect 2016; 5: e10. doi: 10.1038/emi.2016.10

26. Ward MM, Deodhar A, Gensler LS, Dubreuil M, Yu D, Khan MA, et al. 2019 Update of the American College of Rheumatology/Spondylitis Association of America/Spondyloarthritis Research and Treatment Network Recommendations for the Treatment of Ankylosing Spondylitis and Nonradiographic Axial Spondyloarthritis. Arthritis Rheumatol 2019; 71: 1599-613. doi: 10.1002/art.41042

27. Singh JA, Guyatt G, Ogdie A, Gladman DD, Deal C, Deodhar A, et al. 2018 American College of Rheumatology/National Psoriasis Foundation Guideline for the Treatment of Psoriatic Arthritis. Arthritis Care Res (Hoboken) 2019; 71: 2-29. doi: 10.1002/acr.23789

28. Marchesoni A, Olivieri I, Salvarani C, Pipitone N, D'Angelo S, Mathieu A, et al. Recommendations for the use of biologics and other novel drugs in the treatment of psoriatic arthritis: 2017 update from the Italian Society of Rheumatology. Clin Exp Rheumatol 2017; 35: 991-1010.

29. Kalyoncu U, Taşcılar EK, Ertenli Ai, Dalkılıç HE, Bes C, Küçükşahin O, et al. Methodology of a new inflammatory arthritis registry: TReasure. Turk J Med Sci 2018; 48: 856-61. doi: 10.3906/sag-1807-200

30. Aletaha D, Neogi T, Silman AJ, Funovitz J, Felson DT, Bingham CO 3rd, et al. 2010 Rheumatoid arthritis classification criteria: an American College of Rheumatology/European League Against Rheumatism collaborative initiative. Arthritis Rheum 2010; 62: 2569-81. doi: 10.1002/art.27584

Page 9/11 
31. Rudwaleit M, van der Heijde D, Landewe R, Akkoc N, Brandt J, Chou CT, et al. The Assessment of SpondyloArthritis International Society Classification Criteria for Peripheral Spondyloarthritis and for Spondyloarthritis in General. Ann Rheum Dis 2011; 70: 25-31. doi: 10.1136/ard.2010.133645

32. T.C. Sağlık Bakanlığı. Tüberküloz Tanı ve Tedavi Rehberi. Ankara, 2019. [Turkish] https://hsgm.saglik.gov.tr/depo/birimler/tuberkuloz_db/haberler/Tuberkuloz_Tani_Ve_Tedavi_Rehberi_Tuberkuloz_Tani_ve_Tedavi_Rehberi_08.07.2019_Y Accessed 20.10.2020

33. Wong SH, Gao Q, Tsoi KK, Wu WK, Tam LS, Lee N, et al. Effect of immunosuppressive therapy on interferon y release assay for latent tuberculosis screening in patients with autoimmune diseases: a systematic review and meta-analysis. Thorax 2016; 71: 64-72. doi: 10.1136/thoraxjnl-2015-207811

34. Doran MF, Crowson CS, Pond GR, O'Fallon WM, Gabriel SE. Frequency of infection in patients with rheumatoid arthritis compared with controls: a population-based study. Arthritis Rheum 2002; 46: 2287-93. doi: 10.1002/art.10524

35. Glück T, Müller-Ladner U. Vaccination in patients with chronic rheumatic or autoimmune diseases. Clin Infect Dis 2008; 46: 1459-65. doi: 10.1086/587063

36. Meroni PL, Zavaglia D, Girmenia C. Vaccinations in adults with rheumatoid arthritis in an era of new disease-modifying anti-rheumatic drugs. Clin Exp Rheumatol 2018; 36: 317-28. doi: 10.1086/587063

37. Souto A, Maneiro JR, Salgado E, Carmona L, Gomez-Reino JJ. Risk of tuberculosis in patients with chronic immune-mediated inflammatory diseases treated with biologics and tofacitinib: a systematic review and meta-analysis of randomized controlled trials and long-term extension studies. Rheumatology (Oxford) 2014; 53: 1872-85. doi: 10.1093/rheumatology/keu172

38. Duman N, Ersoy-Evans S, Karadag O, Aşçıoğlu S, Sener B, Kiraz S, et al. Screening for Latent Tuberculosis Infection in Psoriasis and Psoriatic Arthritis Patients in a Tuberculosis-Endemic Country: A Comparison of the QuantiFERON@-TB Gold In-Tube Test and Tuberculin Skin Test. Int J Dermatol 2014; 53: 1286-92. doi: 10.1111/ijd.12522

39. Cantini F, Nannini C, Niccoli L, lannone F, Delogu G, Garlaschi G, et al. Guidance for the management of patients with latent tuberculosis infection requiring biologic therapy in rheumatology and dermatology clinical practice. Autoimmun Rev 2015; 14: 503-9. doi: 10.1016/j.autrev.2015.01.011

40. Diel R, Goletti D, Ferrara G, Bothamley G, Cirillo D, Kampmann B, et al. Interferon-ү release assays for the diagnosis of latent Mycobacterium tuberculosis infection: a systematic review and meta-analysis. Eur Respir J 2011; 37: 88-99. doi: 10.1183/09031936.00115110

41. Kim HC, Jo KW, Jung YJ, Yoo B, Lee CK, Kim YG, et al. Diagnosis of latent tuberculosis infection before initiation of anti-tumor necrosis factor therapy using both tuberculin skin test and QuantiFERON-TB Gold In Tube assay. Scand J Infect Dis 2014; 46: 763-9. doi: 10.3109/00365548.2014.938691

42. Lee H, Park HY, Jeon K, Jeong BH, Hwang JW, Lee J, et al. QuantiFERON-TB Gold In-Tube assay for screening arthritis patients for latent tuberculosis infection before starting anti-tumor necrosis factor treatment. PLoS One 2015; 10: e0119260. doi: 10.1371/journal.pone.0119260

43. Soare A, Gheorghiu AM, Aramă V, Bumbăcea D, Dobrotă R, Oneaţă R, et al. Risk of active tuberculosis in patients with inflammatory arthritis receiving TNF inhibitors: a look beyond the baseline tuberculosis screening protocol. Clin Rheumatol 2018; 37: 2391-7. doi: 10.1007/s10067-017-3916-y

\section{Figures}
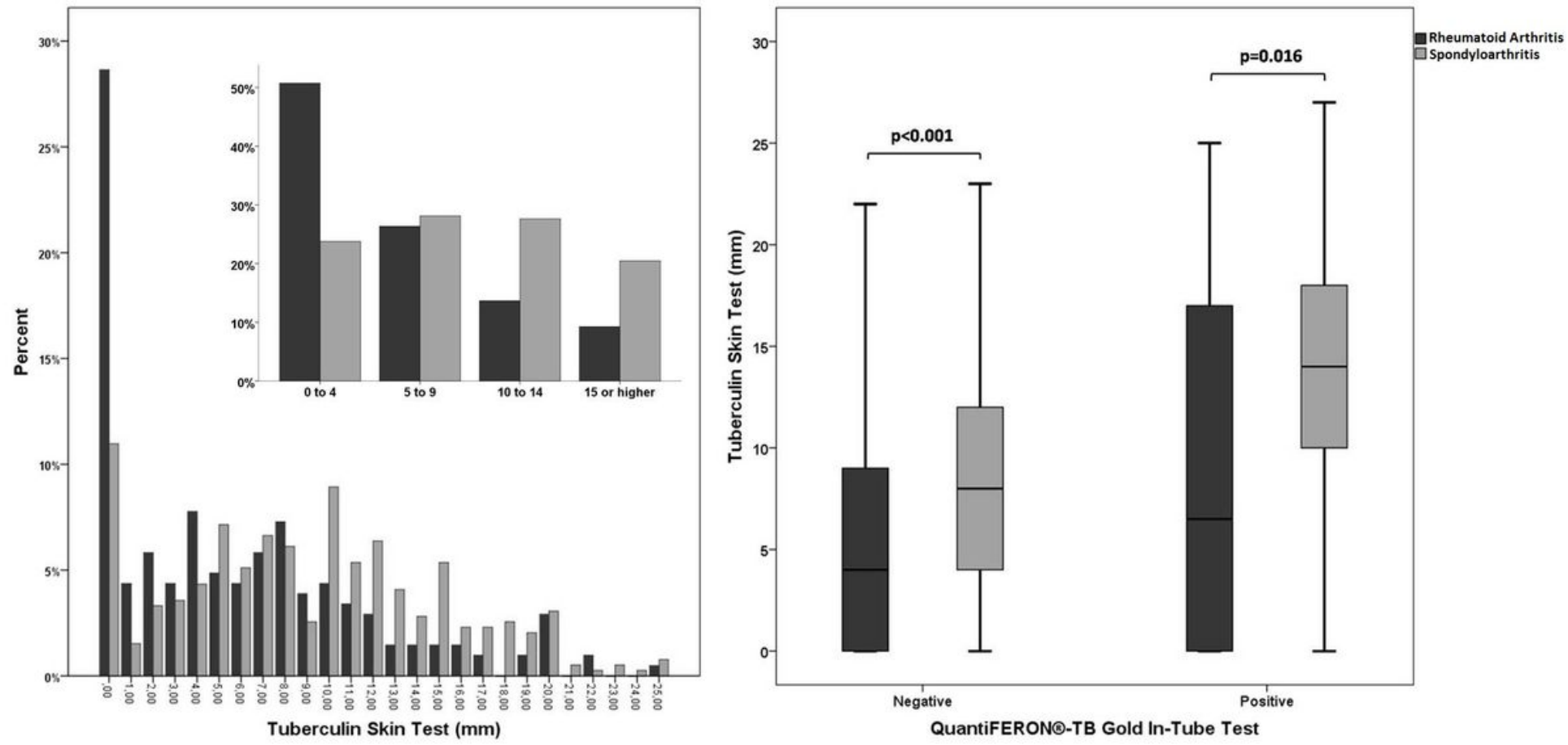

Figure 1

Distribution of TST in rheumatoid arthritis and spondyloarthritis (left) and TST results according to the QFT-GIT status in disease groups (right). 

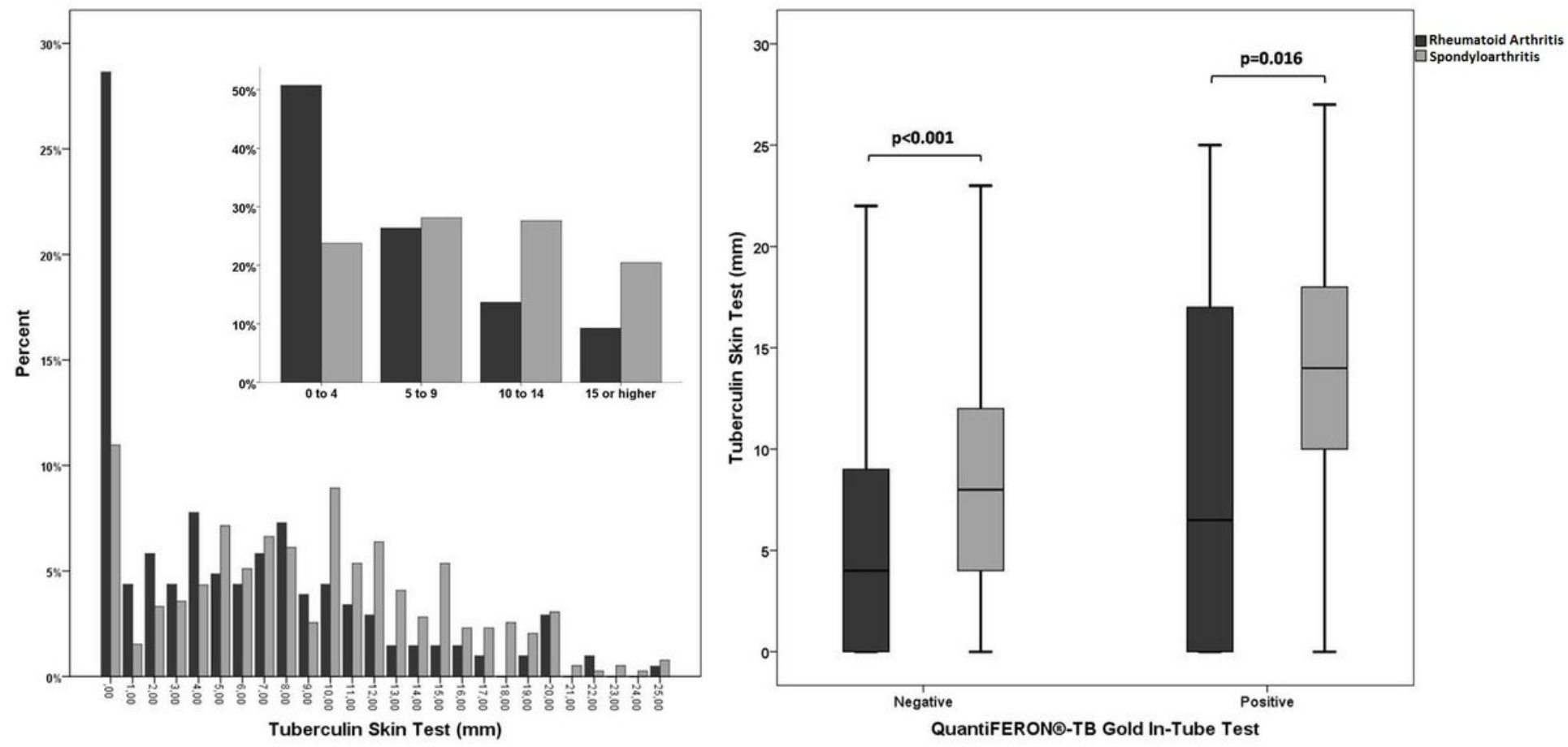

Figure 1

Distribution of TST in rheumatoid arthritis and spondyloarthritis (left) and TST results according to the QFT-GIT status in disease groups (right).

\section{Supplementary Files}

This is a list of supplementary files associated with this preprint. Click to download.

- additionalfile1.docx

- additionalfile1.docx

- additionalfile2.docx

- additionalfile2.docx 